\title{
Myocardial blood flow from SPECT
}

\author{
Piotr Slomka, PhD, ${ }^{a}$ Daniel S. Berman, $M D,{ }^{a}$ and Guido Germano, PhD $^{a}$ \\ a Cedars Sinai Medical Center, Los Angeles, CA
}

Received Dec 18, 2015; accepted Dec 18, 2015

doi:10.1007/s12350-015-0386-y

\section{See related article, pp. 268-277}

\section{WHY DO WE WANT TO MEASURE FLOW?}

Quantification of myocardial blood flow (MBF) at stress and rest in $\mathrm{ml} / \mathrm{g} / \mathrm{min}$ and determination of the myocardial flow reserve (MFR) have long been the domain of PET myocardial perfusion imaging (MPI) with tracers such as ${ }^{15} \mathrm{O}$-Water, ${ }^{13} \mathrm{NH}_{3}$-Ammonia, and more recently ${ }^{82}$ Rubidium. Although at this time, in the routine clinic, PET are currently primarily analyzed visually or at most, semi-quantitatively for the presence and severity of relative perfusion defects using static perfusion images, the MFR measurements can be readily obtained, by rereconstructing the list mode data. This is in particular true for the new 3D PET/CT systems. Thus, the additional quantitative flow measurements can be obtained without additional imaging time or radiation exposure during PET MPI, and therefore are becoming incorporated in the clinical practice. The additional prognostic value of MFR measurements over standard measurement of ischemia has been conclusively demonstrated in two large studies. ${ }^{1,2}$ The potential added diagnostic value of flow measurements for improved prediction of obstructive coronary disease has also been suggested.,

\section{WHY DO WE WANT TO MEASURE FLOW WITH SPECT?}

While blood flow measurements with PET are easy to obtain and well validated, myocardial perfusion SPECT is currently a much more widely used MPI technique clinically. SPECT MPI is also a significantly

\footnotetext{
Reprint requests: Piotr Slomka, Cedars Sinai Medical Center, Los Angeles, CA; slomkap@cshs.org

J Nucl Cardiol 2017;24:278-81.

$1071-3581 / \$ 34.00$

Copyright (c) 2016 American Society of Nuclear Cardiology.
}

cheaper modality. SPECT MPI is still used at least 20 times more often than PET in the routine clinical practice in US, according to the recent estimates. If it were indeed feasible to estimate the MBF and MFR from the standard SPECT MPI scan, it would dramatically enhance the clinical dissemination of these measurements.

\section{IS FLOW MEASUREMENT FEASIBLE ON THE CONVENTIONAL SPECT CAMERAS?}

In this issue of Journal of Nuclear Cardiology, a study by Shershta et $\mathrm{al}^{5}$ analyzes the feasibility results of dynamic flow measurements with ${ }^{99 \mathrm{~m}} \mathrm{Tc}$-tetrofosmin SPECT. Several methodological aspects of this study merit highlighting. The authors studied 16 patients with ${ }^{99 \mathrm{~m}} \mathrm{Tc}$-tetrofosmin SPECT and ${ }^{13} \mathrm{~N}$-ammonia PET studies obtained within 12-month period (mean 3.3 months). They set aside 9 patients from this dataset to "train" the SPECT dynamic quantification by fitting the tracer extraction model to match SPECT and PET results. They subsequently validated the derived SPECT in the remaining 7 cases. The dynamic SPECT scan was performed with a conventional Anger camera with CTbased attenuation correction. To our knowledge, this is the first direct published comparison of SPECT and PET dynamic measurements in humans. Of note, the rotation time of the gantry was 2 minutes. To circumvent this, the authors employed a specialized spatio-temporal reconstruction method developed "in-house," which allowed reconstruction to obtain 5 seconds 3D SPECT frames, despite slow rotation of the gantry. They report the final correlation between the SPECT and PET MBF and MFR values in the 7 patients, which seems to be better for the stress MFF values alone $(r=0.71)$ versus CFR $(r=0.39)$. Notably, however, 95\% limits of agreement computed on a regional basis (from 21 regional samples) were -0.9 to $1.3 \mathrm{ml} / \mathrm{g} / \mathrm{min}$ for $\mathrm{MBF}$ and -3.11 to 2.40 for CFR.

The first impression is that the results are not very encouraging. Very wide limits of agreement as compared to "gold standard" PET flow measurements, 
especially for MFR, certainly would limit clinical applicability. However, when dissecting the methods of this study, it becomes evident that the results are likely overly pessimistic due to several technical limitations, which might lead to potentially better agreement between SPECT and PET flow measurements. Here is why.

The authors develop the model using the SPECTPET flow relationship in only 9 cases. It is likely that the model derived more optimal kinetic model parameters which could be improved with larger number of studies. Further, the time gap between SPECT and PET is rather long (1 to 12 months). Certainly, it will be hard to reproduce the same physiological state in these patients; however, this time difference is not likely the major source of the wide limits of agreement.

As noted, the specialized advanced 4-dimensional reconstruction method developed by the authors can estimate 3D dynamic frames from incomplete projection data and it is very likely that inaccuracies are introduced into the process. The 3D time frames ( 5 seconds) will have only 10 SPECT projections at that time point from a particular limited angle, and it may not be possible to precisely estimate $3 \mathrm{D}$ distribution, since the activity is changing fast in the initial phase. Nevertheless, this technical hurdle could be overcome with newer SPECT hardware. Vendors already offer conventional gantries with rotation time as short as 10 seconds, allowing completed rotation during one $3 \mathrm{D}$ frame, and the feasibility of dynamic SPECT flow data collection has been demonstrated on such systems. ${ }^{6}$ These more rapid frame rates should be sufficient, given that 10 second 3D time frames are routinely used by clinical PET dynamic studies. ${ }^{7,8}$

Another key source of error is the low count rate and associated high Poisson noise with the conventional SPECT camera as compared to PET data, especially for the rest studies (325 MBq injected dose). However, this count limitation could be mitigated by employing fastSPECT systems. At least 3 studies to date have demonstrated the feasibility of the dynamic SPECT kinetic analysis with the new generation solid-state systems, validating results against static perfusion, invasive angiography, and fractional flow reserve measurements (FFR). ${ }^{9-11}$ Not only do these systems allow PET-like acquisition without projections, or at least very fast (3 seconds) detector rotation, but they also offer up to 8 times higher photon sensitivity, approaching the sensitivity of older 2D PET systems. ${ }^{12}$ Thus, dynamic SPECT imaging with these new systems will likely have much lower noise than the results presented in the study by Shershta et al.

\section{ROLL-OFF OF THE UPTAKE RATE VERSUS FLOW CURVE FOR THE ${ }^{99 M}$ TC AGENTS}

A major source of error, which is more difficult to rectify, is the rather flat gradient of the relationship between the flow (in $\mathrm{ml} / \mathrm{g} / \mathrm{min}$ ) and $\mathrm{K} 1$ uptake rate especially at higher flow rates. This so-called "roll-off'" of the uptake curve is related to the poor extraction fraction of the tracer from the blood to the tissue for the currently available $99 \mathrm{mTc}$-based agents, thus resulting in diminished uptake increases with increasing flows. If we use the flow/uptake rate models obtained by Shershta et al, we can see that a two-fold increase in the estimated myocardial blood flow, using the fitted model corresponds to only $\sim 29 \%$ increase of the uptake $(\mathrm{K} 1)$ on the images, as opposed to $\sim 95 \%$ increase in the $\mathrm{K} 1$ uptake rate for the ${ }^{13} \mathrm{NH}_{3}$-ammonia, as depicted in Figure 1. Therefore, even if we were able to find the optimal model for the K1 uptake rate/flow relationship, which provides unbiased estimates, the unavoidable effect would be at least 3 times amplification of the random error of the flow estimates.

In a recent study by Wells et al, dynamic SPECT was studied for 3 different tracers ${ }^{201} \mathrm{Tl}$, ${ }^{99 \mathrm{~m}} \mathrm{Tc}$-tetrofosmin, and ${ }^{99 m}$ Tc-sestamibi with the fast-SPECT solidstate camera with CT attenuation correction. ${ }^{9}$ Similar methods for the flow correction were used but the data were calibrated to the microsphere results obtained in animal experiments rather than to PET. In that study, Wells et al show similar roll-off of the extraction curve

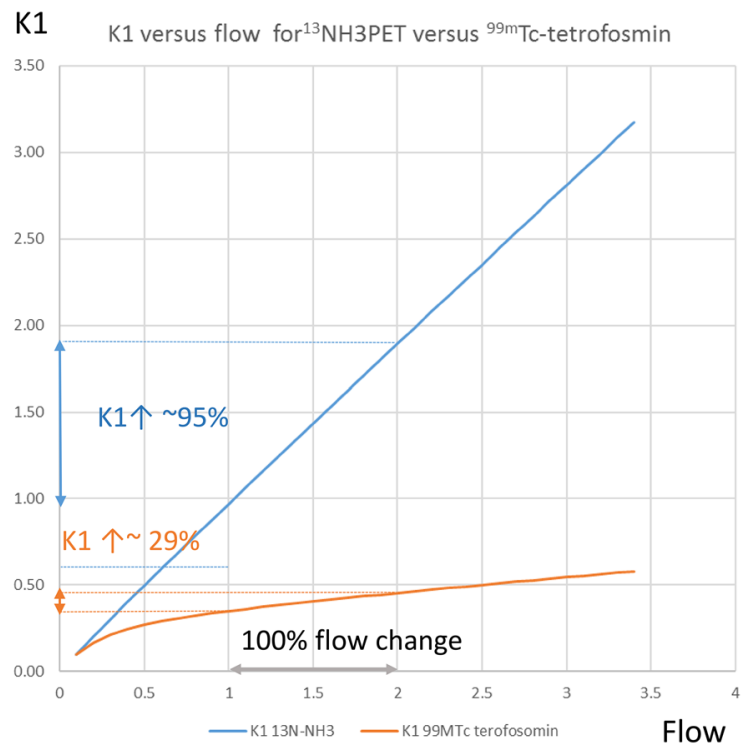

Figure 1. Relationship between uptake rate and flow for the ${ }^{13} \mathrm{NH}_{3}$-ammonia and ${ }^{99 \mathrm{~m}}$ Tetrofosmin according to the models derived by Shershta et al. 
to the one shown by Shershta et al. The roll-off for ${ }^{201} \mathrm{Tl}$ was less pronounced, but on the other hand, the ${ }^{201} \mathrm{Tl}$ images were noisier due to lower injected dose of this tracer, resulting in similar final signal-to noise ratio in the MBF measurements. Thus, it seems that although the currently available ${ }^{99 \mathrm{~m}} \mathrm{Tc}$-based agents are suboptimal due to extraction fraction, it is not clear whether other SPECT tracers will provide better solution. Thus, precisely estimating the differences between stress and rest is more difficult for the available ${ }^{99 \mathrm{~m}} \mathrm{Tc}$-based tracer than for tracers such as ${ }^{13} \mathrm{~N}$-ammonia with higher extraction rate, and count limitations are presented by ${ }^{201} \mathrm{Tl}$.

One possible solution to the extraction fraction of the available ${ }^{99 \mathrm{~m}} \mathrm{Tc}$ tracers might be potentially available through the use of ${ }^{99 \mathrm{~m}} \mathrm{Tc}$ teboroxime. This agent has been shown to have uptake versus flow characteristics that are similar to those of ${ }^{13} \mathrm{NH}_{3}$-ammonia. Rapid washout from the myocardium and rapid liver uptake were the limitations of the tracer, requiring very fast acquisitions for clinical use, ${ }^{13}$ leading to low utilization and its discontinuation. Nevertheless, with the new SPECT systems, rapid SPECT acquisitions of MPI studies could be more feasible. The accuracy of the standard perfusion and function evaluation with ${ }^{99 \mathrm{~m}} \mathrm{Tc}$ teboroxime on the new generation fast MPS systems would have to be rigorously compared to that of currently used ${ }^{99 \mathrm{~m}} \mathrm{Tc}$ tracers to ensure that the basic well-established basic utility of SPECT MPI is preserved.

\section{MBF OR MFR?}

In the study by Shershta et al, the PET-SPECT MFR correlations were found to be poorer than the correlations between stress PET and stress SPECT MBF. Perhaps, we could consider stress-only MBF measurements? We would not have to worry about low restinjected dose and its effect on high MFR variability. Additionally, it is estimated that up to $60 \%$ of SPECT scans could be performed as stress-only, canceling rest scan. ${ }^{14}$ Perhaps an additional stress flow measurement would be particularly valuable in these cases, confirming normal scan? At least 2 large PET studies have compared the diagnostic value of the hyperemic stress flow versus MFR finding that stress MBF flow alone provides superior accuracy to MFR. ${ }^{15,16}$ Therefore, estimation of the stress flow from a single scan may prove to be a very attractive approach, removing the technical difficulties associated with lower resting dose and potentially even increasing the application of stressonly procedures.

\section{SUMMARY AND FUTURE PERSPECTIVES}

Despite rather large discrepancies between PET and SPECT dynamic flow quantification reported by Shershta et al and despite intrinsic limitations of the ${ }^{99 \mathrm{~m}} \mathrm{Tc}$ based tracers, we believe that SPECT myocardial blood flow measurements will find their place in the clinical practice. From the practical standpoint, the standard SPECT acquisition protocol will have to be modified to allow early SPECT acquisition immediately after injection followed by the standard perfusion scan at a later time. However, other than this need for modification of the protocol, there are no major technical limitations. The data can be obtained without additional radiation dose and can be rapidly processed by highly automated software. It is likely that fast MPS will be used for this purpose, improving count statistics and allowing fast 3D frame rate. It is possible that stress-only flow measurements will be easier to obtain and will actually correlate better with PET measurements. With current Tc-99m tracers, it is likely that it will be much more difficult to estimate subtle flow changes with SPECT than with PET. However, even if the quantitative agreement of dynamic SPECT with PET measurements is moderate with current SPECT tracers, it is possible that gross flow "estimates" by SPECT or even a dichotomous normal/ abnormal flow categorization will find some clinical application, allowing to reclassify patient risk and help in certain diagnostic situations. Larger diagnostic and prognostic SPECT studies should be conducted to validate these claims.

\section{Disclosure}

Cedars-Sinai Medical Center receives royalties for the quantitative assessment of function, perfusion, and flow, a portion of which is distributed to the authors.

\section{References}

1. Murthy VL, Naya M, Foster CR, Hainer J, Gaber M, Di Carli G, et al. Improved cardiac risk assessment with noninvasive measures of coronary flow reserve. Circulation 2011;124:2215-24.

2. Ziadi MC, Dekemp RA, Williams KA, Chow BJ, Renaud JM, Ruddy $\mathrm{T}$, et al. Impaired myocardial flow reserve on rubidium- 82 positron emission tomography imaging predicts adverse outcomes in patients assessed for myocardial ischemia. J Am Coll Cardiol 2011;58:740-8.

3. Ziadi MC, Dekemp RA, Williams K, Renaud JM, Chow BJ, Klien R, et al. Does quantification of myocardial flow reserve using rubidium82 positron emission tomography facilitate detection of multivessel coronary artery disease? J Nucl Cardiol 2012;19:670-80.

4. Naya M, Murthy VL, Taqueti VR, Foster CR, Klein J, Garber M, et al. Preserved coronary flow reserve effectively excludes high- 
risk coronary artery disease on angiography. J Nucl Med 2014;55:248-55.

5. Shrestha U, Sciammarella M, Alhassen F, Yeghiazarians Y, Ellin $\mathrm{J}$, Verdin E, et al. Measurement of absolute myocardial blood flow in humans using dynamic cardiac SPECT and 99mtc-tetrofosmin: Method and validation. J Nucl Cardiol 2015. doi:10.1007/ s12350-015-0320-3.

6. Klein R, Hung G, Wu T, Huang WS, Li D, Hsu B. Feasibility and operator variability of myocardial blood flow and reserve measurements with 99mtc-sestamibi quantitative dynamic SPECT/CT imaging. J Nucl Cardiol 2014;21:1075-88.

7. Slomka PJ, Alexanderson E, Jacome R, Jiménez M, Romero E, Meave A, et al. Comparison of clinical tools for measurements of regional stress and rest myocardial blood flow assessed with 13nammonia PET/CT. J Nucl Med 2012;53:171-81.

8. Dekemp RA, Declerck J, Klein R, Nakazato R, Tonge C, Arumugam $P$, et al. Multisoftware reproducibility study of stress and rest myocardial blood flow assessed with $3 \mathrm{~d}$ dynamic PET/CT and a 1-tissue-compartment model of $82 \mathrm{rb}$ kinetics. J Nucl Med 2013;54:571-7.

9. Wells RG, Timmins R, Klein R, Lockwood J, Marvin B, Wei L, et al. Dynamic SPECT measurement of absolute myocardial blood flow in a porcine model. J Nucl Med 2014;55:1685-91.

10. Ben-Haim S, Murthy VL, Breault C, Allie R, Sitek A, Roth N, et al. Quantification of myocardial perfusion reserve using dynamic SPECT imaging in humans: A feasibility study. J Nucl Med 2013;54:873-9.

11. Bouallegue FB, Roubille F, Lattuca B, Cung TT, Macia JC, Gervasoni $\mathrm{R}$, et al. SPECT myocardial perfusion reserve in patients with multivessel coronary disease: Correlation with angiographic findings and invasive fractional flow reserve measurements. J Nucl Med 2015;56:1712-7.

12. Slomka PJ, Pan T, Berman DS, Germano G. Advances in SPECT and PET hardware. Prog Cardiovasc Dis. 2015;57:566-78.

13. Chua T, Kiat H, Germano G, et al. Rapid back to back adenosine stress/rest technetium-99m teboroxime myocardial perfusion SPECT using a triple-detector camera. J Nucl Med 1993;34:148593.

14. Chang SM, Nabi F, Xu J, Raza U, Mahmarian JJ. Normal stressonly versus standard stress/rest myocardial perfusion imaging: Similar patient mortality with reduced radiation exposure. J Am Coll Cardiol 2010;55:221-30.

15. Danad I, Raijmakers PG, Appelman YE, Harms HJ, de Haan S, van den Oever ML, et al. Hybrid imaging using quantitative h215o PET and CT-based coronary angiography for the detection of coronary artery disease. J Nucl Med 2013;54:55-63.

16. Kajander S, Saraste M, Pietilä M, Ukkonen H, Saraste A, Sipilä HT, et al. Cardiac positron emission tomography/computed tomography imaging accurately detects anatomically and functionally significant coronary artery disease. Circulation 2010;122:603-13. 